\title{
A NON-PLANAR BOUNDARY PROBLEM FOR THE WAVE EQUATION*
}

\author{
BY \\ GEORGE K. MORIKAWA \\ (Hughes Aircraft Company, Research and Development Laboratories)
}

1. Introduction. Notwithstanding the relatively recent outburst of activity on the part of aerodynamicists concerning problems dealing with the wave-equation, some problems with radiation type conditions still deserve attention. An important class of problems of this type are those with non-planar, non-axially-symmetric boundaries, in particular, wing-body problems in linearized supersonic flow. For slender wing-bodies [1], where variations in the flow direction are small, the stationary three-dimensional problem is reduced to a two-dimensional potential (Laplace) problem in the cross-flow plane. For conical wing-bodies [2], where the surface is generated by linear elements passing through the vertex, a reduction from three to two-dimensions is evident, and a transformation (Chaplygin) further reduces the subsequent elliptic problem to a twodimensional potential problem. However, for more general type boundaries, the problem is relatively difficult. One of the first attempts in this direction has been made by Ferrari [3], using an iteration procedure which is also discussed by Lagerstrom and Van Dyke [4]. In this paper the problem, which the author considers to be the fundamental wing-body problem for stationary linearized supersonic flow, is discussed. An approximate solution is obtained and expressed in terms of the pressure, a quantity of interest to aerodynamicists.

2. Formulation of the problem. Consider the wing-body configuration shown in Figure 1 , where the body is an infinitely long, circular cylinder (normalized to radius =1) and the wing is a semi-infinite flat plate with the leading edge normal to the free-stream direction defined by the velocity $W$, i.e., zero sweep-back. $W$ is the uniform flow (nominally in the $z$-direction) far upstream and away from the body and the dashed lines from the leading edge-body junction are elements of Mach cones indicating supersonic leading edges. The problem considered here is the case where both body and wing are at a small angle of attack, $\alpha$, with respect to the uniform flow at $-\infty$; the more general case, where the body is at $\alpha$ and the wing has an arbitrary angle of attack distribution, can be handled as easily. Since the leading edges of the wing are supersonic, the flow field upstream is known everywhere and the boundary conditions are of the first kind [5], where one component of the velocity, namely, $v \equiv \varphi_{\nu}$, is known everywhere in the plane of the wing ( $x, z$ plane) and on the body. By a simple super-position procedure made possible by the linearization, the original problem with both body and wing at $\alpha$

${ }^{*}$ Received July 19, 1951. This paper is part of a thesis submitted in partial fulfillment of the requirements for the degree of Doctor of Philosophy at California Institute of Technology, June, 1949, and a subsequent report, "The Wing-Body Problem for Linearized Supersonic Flow", Jet Propulsion Laboratory, California Institute of Technology, Progress Report No. 4-116 (Dec. 19, 1949), written under U. S. Army Ordnance Contract No. W-04-200-ORD-455. A more complete description of the physical problem (and related aspects) than given in this paper is presented in the report, hereafter referred to as the "JPL report". The author wishes to thank Professors H. J. Stewart, P. A. Lagerstrom, A. Erdelyi and C. R. DePrima for their suggestions and criticisms. 
can be replaced by an equivalent problem* with body at zero angle and wing at angle of attack $\alpha$, plus an apparent twist imposed by the original flow upstream of the leading

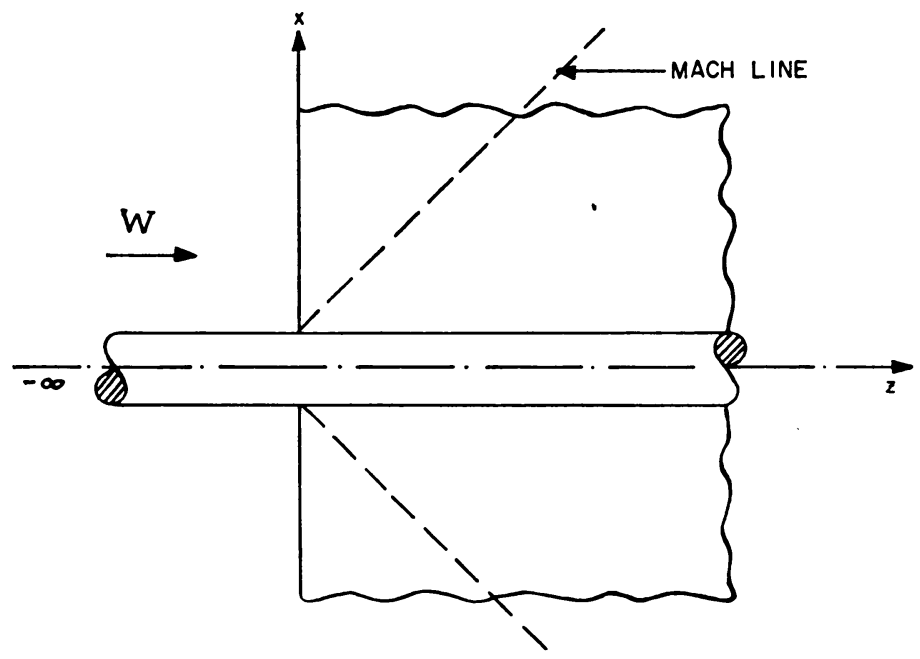

Fig. 1. Wing-body Configuration.

edge. Then the boundary conditions in cylindrical polar coordinates, in terms of the perturbation velocity potential, are:

$$
\frac{1}{r} \varphi_{\theta}(r, 0, z)=-W \alpha\left(1+\frac{1}{r^{2}}\right), \quad z>0, \quad \varphi_{r}(1, \theta, z)=0
$$

where $\varphi$ satisfies the wave equation

$$
\Delta \varphi-\varphi_{z z} \equiv \varphi_{r r}+\frac{1}{r} \varphi_{r}+\frac{1}{r^{2}} \varphi_{\theta \theta}-\varphi_{z z}=0 .
$$

For arbitrary Mach number $M$, the coefficient of $\varphi_{z z}$ is $-\left(M^{2}-1\right)$, but there is no loss in generality in taking $M=2^{1 / 2}$, since, by a simple similarity transformation [5] (linear), the arbitrary $M$ case results. The initial condition ( $z$ is a time-like direction) for the equivalent problem is taken to be

$$
\varphi(r, \theta, 0+)=\varphi_{z}(r, \theta, 0+)=0 .
$$

Actually, $\varphi_{z}(r, 0,0+) \neq 0$, that is, $\varphi_{z} \neq 0$ if $z=0$ is approached from positive $z$ in the $x, z$ plane, but this condition causes no difficulty here.** Then this radiation type problem given by Equations (1a), (1b) and (1c) is a natural problem for the methods of the Laplace transformation; $\dagger$ and subsequently it will be evident that these methods lead to an approximate solution of an inherently complicated problem.

*Actually, the equivalent problem consists of two problems, the second consisting of the body alone at angle of attack $\alpha$; but since the pressure component $\varphi_{z}$ is the quantity of usual interest, the second problem contributes nothing.

${ }^{* *}$ A necessary condition for uniqueness is the condition of outgoing waves.

†Several British workers have recently applied Laplace transform methods to supersonic flow problems; see, e.g. $[6,7]$. 
Assuming that the solution is twice differentiable and that the second derivatives have Laplace transforms, the equivalent problem given by Eqs. (1) becomes the transformed problem

$$
\Delta \psi-s^{2} \psi=0
$$

with the boundary conditions

$$
\frac{1}{r} \psi_{\theta}(r, 0 ; s)=-\frac{W \alpha}{s}\left(1+\frac{1}{r^{2}}\right), \quad \psi_{r}(1, \theta ; s)=0,
$$

where

$$
\psi(r, \theta ; s)=\int_{0}^{\infty} e^{-s z} \varphi(r, \theta, z) d z
$$

is the Laplace transform of $\varphi$ with respect to the free-stream direction, $z$, and $s$ is the transform variable. (A convenient notation is to write $\psi=\mathscr{L}\{\varphi ; s\}$ and the inverse Laplace transform $\varphi=\mathscr{L}^{-1}\{\psi ; z\}$; then the pressure $p=-\rho W \varphi_{z}$, where $\rho$ is the freestream density and $\left.\varphi_{z}=\mathscr{L}^{-1}\{s \psi ; z\}\right)$. For convenience in presentation, since none of the essential features are lost, the sweepback is taken equal to zero. The differential equation (Eq. 2a) is the two-dimensional modified Helmholtz equation with the parameter $s(\operatorname{Re} s>0)$ and the transformed problem is an elliptic boundary-value problem in the $r, \theta$ plane. For strong conditions $[8,9]$ on $\psi$ exterior and on the boundary of the region, i.e., $\psi$ is twice continuously differentiable in $(r, \theta)$, the solution is expressible as an integral in terms of $\psi$ and the outward normal derivative $\psi_{n}$ on the boundary by application of the Green's theorems. But $\psi$ and $\psi_{n}$ are related on the boundary $C$ and the solution takes the form

$$
\psi(r, \theta ; s)=-\frac{1}{2 \pi} \int_{c}\left(\psi \frac{\partial G}{\partial n}-G \frac{\partial \psi}{\partial n}\right) d l,
$$

where $G$ is the sum of a singular and a regular function in the region. If $G$ is chosen such that $\partial G / \partial n=0$ on $C$

$$
\psi(r, \theta ; s)=\frac{1}{2 \pi} \int_{C} G \frac{\partial \psi}{\partial n} d l,
$$

where $G$ is called the second Green's function. The Green's function is then characterized by the following conditions:

1) $G\left(r, \theta ; r^{\prime}, \theta^{\prime}\right) \equiv G(P ; Q)$ satisfies Equation (2a) for $P(r, \theta) \neq Q\left(r^{\prime}, \theta^{\prime}\right)$;

2) $G\left(r, \theta ; r^{\prime}, \theta^{\prime}\right)$ has the proper singularity at $P(r, \theta)=Q\left(r^{\prime}, \theta^{\prime}\right)$;

3) $\frac{\partial G}{\partial n}=0$ on $C$.

A consequence of the first two conditions is that $G\left(r, \theta ; r^{\prime}, \theta^{\prime}\right)=G\left(r^{\prime}, \theta^{\prime} ; r, \theta\right)$ symmetric in $(P ; Q)$. Weaker conditions, e.g., the condition that $\psi_{n}$ may be discontinuous, but integrable on the boundary, are permissible for Eq. (3b) as in potential theory [10].

The fundamental solution of the modified Helmholtz equation (Eq. 3b), i.e., the solution independent of $\theta$ and singular at the origin, is $K_{0}(s r)$, the modified Bessel func- 
tion of the second kind of zero order [11]. For a fixed point $P(r, \theta)$ with a variable point $Q\left(r^{\prime}, \theta^{\prime}\right)$, the fundamental solution may be written immediately as $K_{0}(s \rho)$ since the differential equation is invariant under translation; $\rho=\left[r^{2}+r^{\prime 2}-2 r r^{\prime} \cos \left(\theta-\theta^{\prime}\right)\right]^{1 / 2}$ is the distance between $P$ and $Q$. This solution may be interpreted as the Green's function for the entire $x, y$ plane, since it satisfies the required properties (Eq. 4).

In determining the Green's function for a region with a given boundary, the function sought will always have the form

$$
G(P ; Q)=K_{0}(s \rho)+h(P ; Q),
$$

where $h(\rho ; Q)$ is regular in the region. Since $K_{0}(s \rho)$ is symmetric in $(P ; Q)$ then $h(P ; Q)$ must also be. The invariance properties of the differential equation will be helpful in determining $h(P ; Q)$ and give some intuitive meaning to it. The Helmholtz equation (Eq. 2a) can easily be verified to be invariant under the following transformations:

1) translation, $(\bar{x}, \bar{y})=(x+a, y+b)$, where $a$ and $b$ are constants;

2) reflection on the axes, e.g., on the $x$-axis, $(\bar{x}, \bar{y})=(x,-y)$ or reflection on any straight line;

3) rotation, $(\bar{r}, \bar{\theta})=(r, \theta+c)$ where $c$ is a constant.

There appears to be no simple invariant transformation with respect to inversion on the unit circle, as there is for Laplace's equation. Such a transformation would be helpful in obtaining an intuitive notion on constructing the Green's function for the circle [12].

For the transformed wing-body problem given by Eqs. (2) the solution can be confined to the upper half-plane and the complete solution obtained later by using the known symmetry conditions on the solution with respect to the $x, z$ plane. Then the boundary is that given in Fig. 2. The Green's function for the upper half-plane is

$$
G_{1}(P ; Q)=K_{0}(s \rho)+K_{0}\left(s \rho_{1}\right)
$$

where

$$
\rho_{1}=\left[r^{2}+{r^{\prime}}^{2}-2 r r^{\prime} \cos \left(\theta+\theta^{\prime}\right)\right]^{1 / 2}
$$

is the distance between $P$ and the reflection of $Q$ on the $x$-axis. The Green's function for the unit circle is easily obtained by using the addition theorem [11] for $K_{0}(s \rho)$ :

$$
K_{0}(s \rho)=I_{0}(s r) K_{0}\left(s r^{\prime}\right)+2 \sum_{n=1}^{\infty} I_{n}(s r) K_{n}\left(s r^{\prime}\right) \cos \left[n\left(\theta-\theta^{\prime}\right)\right], \quad r<r^{\prime}
$$

with $r$ and $r^{\prime}$ interchanged for $r>r^{\prime}$, where $I_{n}$ and $K_{n}$ are modified Bessel functions of the first and second kinds, respectively, of the $n$th order.

For $r<r^{\prime}$ consider $h(P ; Q)$ of the form

$$
h(P ; Q)=A_{0} K_{0}(s r)+\sum_{n=1}^{\infty} A_{n} K_{n}(s r) \cos \left[n\left(\theta-\theta^{\prime}\right)\right],
$$

where $A_{0}$ and $A_{n}$ are determined to satisfy the boundary condition

$$
\left.\frac{\partial G_{2}(P ; Q)}{\partial r}\right|_{r=1}=0
$$


then

$$
A_{0}=-\frac{I_{0}^{\prime}(s)}{K_{0}^{\prime}(s)} K_{0}\left(s r^{\prime}\right), \quad A_{n}=-2 \frac{I_{n}^{\prime}(s)}{K_{n}^{\prime}(s)} K_{n}\left(s r^{\prime}\right),
$$

where the primes on the Bessel functions denote differentiation with respect to $r$ and

$$
\begin{aligned}
G_{2}(P ; Q)=K_{0}\left(s r^{\prime}\right) & {\left[I_{0}(s r)-\frac{I_{0}^{\prime}(s)}{K_{0}^{\prime}(s)} K_{0}(s r)\right] } \\
& +2 \sum_{n=1}^{\infty} K_{n}\left(s r^{\prime}\right)\left[I_{n}(s r)-\frac{I_{n}^{\prime}(s)}{K_{n}^{\prime}(s)} K_{n}(s r)\right] \cos \left[n\left(\theta-\theta^{\prime}\right)\right], \quad r<r^{\prime}
\end{aligned}
$$

with $r$ and $r^{\prime}$ interchanged for $r>r^{\prime}$.

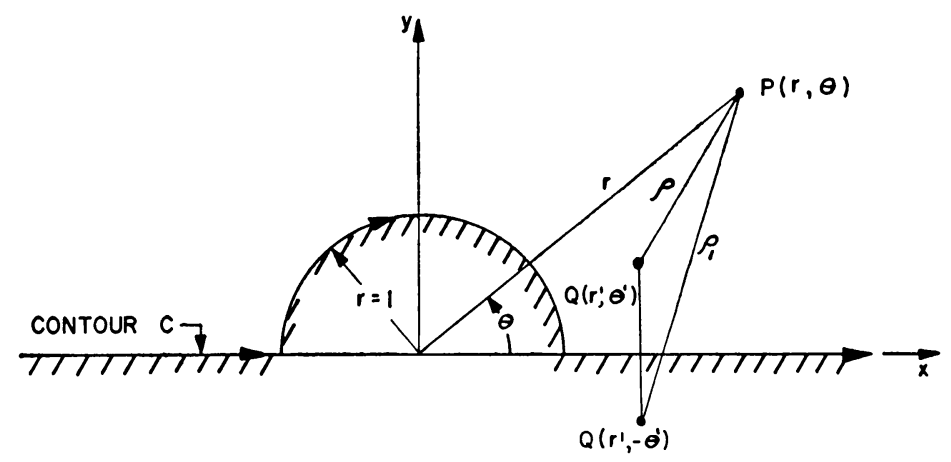

Fig. 2. Boundary for Transformed Problem.

The Green's function for the boundary given in Fig. 2 is obtained simply by using the reflection property on the $x$-axis with the results for the circle (Eq. 7e) and

$$
\begin{aligned}
G(P ; Q)=2 K_{0}\left(s r^{\prime}\right) & {\left[I_{0}(s r)-\frac{I_{0}^{\prime}(s)}{K_{0}^{\prime}(s)} K_{0}(s r)\right] } \\
& +4 \sum_{n=1}^{\infty} K_{n}\left(s r^{\prime}\right)\left[I_{n}(s r)-\frac{I_{n}^{\prime}(s)}{K_{n}^{\prime}(s)} K_{n}(s r)\right] \cos n \theta \cos n \theta^{\prime}, \quad r<r^{\prime}
\end{aligned}
$$

with $r$ and $r^{\prime}$ interchanged for $r>r^{\prime}$. A convenient equivalent form to Eq. (8a) is

$$
\begin{aligned}
G(P ; Q)=K_{0}(s \rho)+K_{0}\left(s \rho_{1}\right)-2\left[\frac{I_{0}^{\prime}(s)}{K_{0}^{\prime}(s)} K_{0}(s r) K_{0}\left(s r^{\prime}\right)\right. & \\
& \left.+2 \sum_{n=1}^{\infty} \frac{I_{n}^{\prime}(s)}{K_{n}^{\prime}(s)} K_{n}(s r) K_{n}\left(s r^{\prime}\right) \cos n \theta \cos n \theta^{\prime}\right]
\end{aligned}
$$

With the determination of the Green's function the transformed problem is formally solved. There follows, for each solution of specific problems, the interpretation by the inverse Laplace transformation into the solution of the original physical problem; sometimes this is a formidable task.

Some intuitive meaning may be given to the Green's functions for the transformed problem. For example, the inverse Laplace transform of the fundamental solution $K_{0}(s \rho)$ represents a supersonic source singularity in the unobstructed physical space. 
3. Splitting of the transformed solution $\psi$. Since on the circle $\psi_{n}=-\psi_{r}=0$, the transformed solution by Eqs. (2b) and (3b) is

$$
\psi(r, \theta ; s)=\frac{W \alpha}{2 \pi s} \int_{1}^{\infty}\left(1+\frac{1}{r^{\prime 2}}\right)\left[G\left(r, \theta ; r^{\prime}, \pi\right)+G\left(r, \theta ; r^{\prime}, 0\right)\right] d r^{\prime} .
$$

Using the second form of the Green's function (Eq. 8b)

$$
\begin{aligned}
\psi(r, \theta ; s) & =\frac{W \alpha}{\pi s} \int_{1}^{\infty}\left(1+\frac{1}{r^{\prime 2}}\right)\left\{K_{0}\left[s\left(r^{2}+{r^{\prime}}^{2}-2 r r^{\prime} \cos \theta\right)^{1 / 2}\right]\right. \\
& \left.+K_{0}\left[s\left(r^{2}+{r^{\prime}}^{2}+2 r r^{\prime} \cos \theta^{\prime}\right)^{1 / 2}\right]\right\} d r^{\prime}-\frac{2 W \alpha}{\pi s} \int_{1}^{\infty}\left(1+\frac{1}{r^{\prime 2}}\right)\left[\frac{I_{0}^{\prime}(s)}{K_{0}^{\prime}(s)} K_{0}(s r) K_{0}\left(s r^{\prime}\right)\right. \\
& \left.+2 \sum_{n=1}^{\infty} \frac{I_{2 n}^{\prime}(s)}{K_{2 n}^{\prime}(s)} K_{2 n}(s r) K_{2 n}\left(s r^{\prime}\right) \cos 2 n \theta\right] d r^{\prime} \\
& \equiv \psi^{(1)}+\psi^{(2)}
\end{aligned}
$$

This transformed solution is confined to the first quadrant; the solution in the other quadrants are obtained by symmetry arguments. The solution given by Eq. (9b) is written as two integrals, $\psi^{(1)}$ and $\psi^{(2)}$, respectively, for two reasons: 1) each can be given a physical interpretation, and 2) the difficult core of the solution, i.e., the second integral $\psi^{(2)}$, is separated from $\psi^{(1)}$, the inverse Laplace transform of which can be obtained simply. By inspection, it is evident that $\psi^{(1)}$ can be regarded as the transformed solution for the equivalent problem (cf. first paragraph of Sec. 2) where the circular cylinder $(\alpha=0)$ is replaced by a flat plate $(\alpha=0)$; thus $\psi^{(1)}$ is called the "flat plate" solution. Then the second part of the solution $\psi^{(2)}$ can be interpreted as the correction solution needed to satisfy the boundary condition of zero flow through the circular cylinder, i.e., the circular cylinder is a stream tube; thus $\psi^{(2)}$ is called the "body" solution. The principal difficulties arise in attempting to perform the inverse Laplace transformation of $\psi^{(2)}$.

4. The "flat plate" solution $\varphi^{(1)}$. The "flat plate" solution in terms of the pressure component (cf. foot note following Eq. 2c) $\varphi_{z}^{(1)}$ on the surface is conveniently written in Cartesian Coordinates. The transformed solution for $\varphi_{z}^{(1)}=\mathcal{L}^{-1}\left\{s \psi^{(1)} ; z\right\}$ by Eq. (9b) is

$$
\begin{aligned}
s \psi^{(1)}(x, y ; s)=\frac{W \alpha}{\pi} \int_{1}^{\infty}\left(1+\frac{1}{\xi^{2}}\right)\left\{K _ { 0 } \left[s \left((x-\xi)^{2}+\right.\right.\right. & \left.\left.y^{2}\right)^{1 / 2}\right] \\
& \left.+K_{0}\left[s\left((x+\xi)^{2}+y^{2}\right)^{1 / 2}\right]\right\} d \xi .
\end{aligned}
$$

The order of integration can be interchanged and the inverse Laplace transformation is performed first:

$$
\begin{aligned}
& \mathcal{L}^{-1}\left\{K_{0}\left[s\left((x-\xi)^{2}+y^{2}\right)^{1 / 2}\right] ; z\right\} \\
& \quad=0, \quad \xi<\left[x-\left(z^{2}-y^{2}\right)^{1 / 2}\right] \quad \text { and } \quad\left[x+\left(z^{2}-y^{2}\right)^{1 / 2}\right]<\xi \\
& \quad=\left[z^{2}-y^{2}-(x-\xi)^{2}\right]^{-1 / 2}, \quad\left[x-\left(z^{2}-y^{2}\right)^{1 / 2}\right]<\xi<\left[x+\left(z^{2}-y^{2}\right)^{1 / 2}\right]
\end{aligned}
$$


and

$$
\begin{aligned}
\mathscr{L}^{-1}\left\{K _ { 0 } \left[s \left((x+\xi)^{2}\right.\right.\right. & \left.\left.\left.+y^{2}\right)^{1 / 2}\right] ; z\right\} \\
& =0, \quad\left[\left(z^{2}-y^{2}\right)^{1 / 2}-x\right]<\xi \\
& =\left[z^{2}-y^{2}-(x+\xi)^{2}\right]^{-1 / 2}, \quad 0<\xi<\left[\left(z^{2}-y^{2}\right)^{1 / 2}-x\right]
\end{aligned}
$$

In addition, since the lower limit of the integral is 1 , three regions of integration are

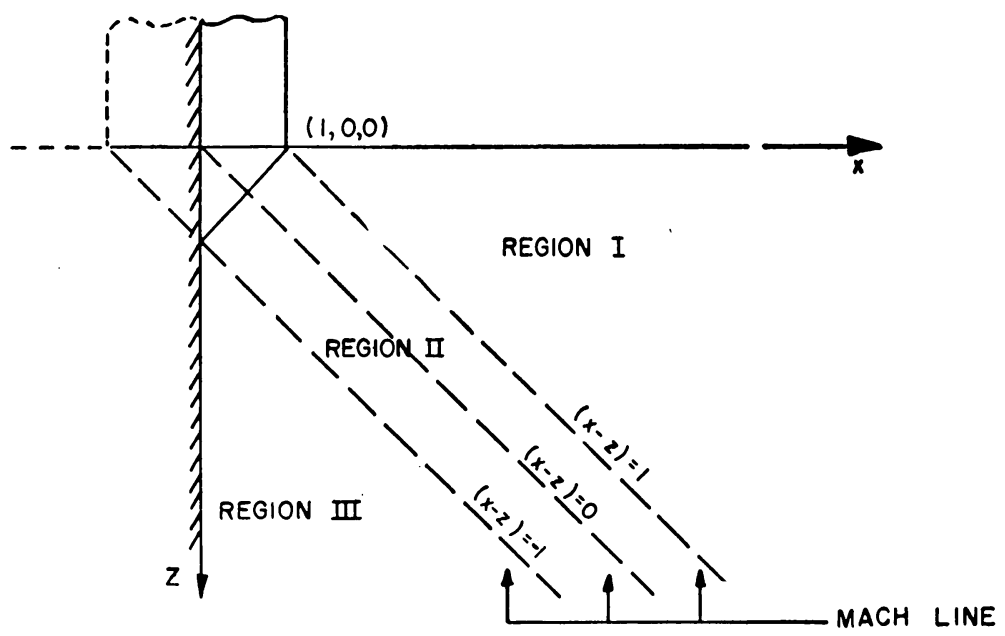

FIG. 3. Regions of Influence in the $x, z$ Plane.

defined and have a physical interpretation (cf. Fig. 3, where these regions are shown in the $x, z$ plane):

Region I, $(x-z)>1$ : Region of influence of the Mach cones from the wing leading edge

Region II, $-1<(x-z)<1$ : Region of influence of the Mach cone from the wing leading edge-body junction plus Region I.

Region III, $(x-z)<-1$ : Region of influence of the Mach cones from the opposite wing leading edge plus Region I plus Region II.

The "flat plate" solution, $\varphi_{z}^{(1)}$, on the wing surface $(x, z$ plane) in these three regions is given in terms of elementary functions (cf. JPL report) and is shown graphically in Fig. 4 by dashed curves. For the chordwise pressure distribution (Fig. $4 \mathrm{~b}$ ) attention is focused primarily on the body (a fairly complete description of the solutions is given in the JPL report).

An additional solution which can be obtained immediately is important in the discussion of the complete solution. The "flat plate" configuration (cf. Sec. 3) is modified now by inserting a semi-infinite plane barrier at $x=1$, parallel to the $y, z$ plane downstream from the leading edge-body junction. This modification does not affect the solution $\varphi^{(1)}$ outside the Mach cone from the leading edge-body junction; but inside 
this region of influence for $x \geq 1$, the inserted plane simulates the limiting case of a body with infinite radius. Thus, in this region, the solution (called the "modified flat

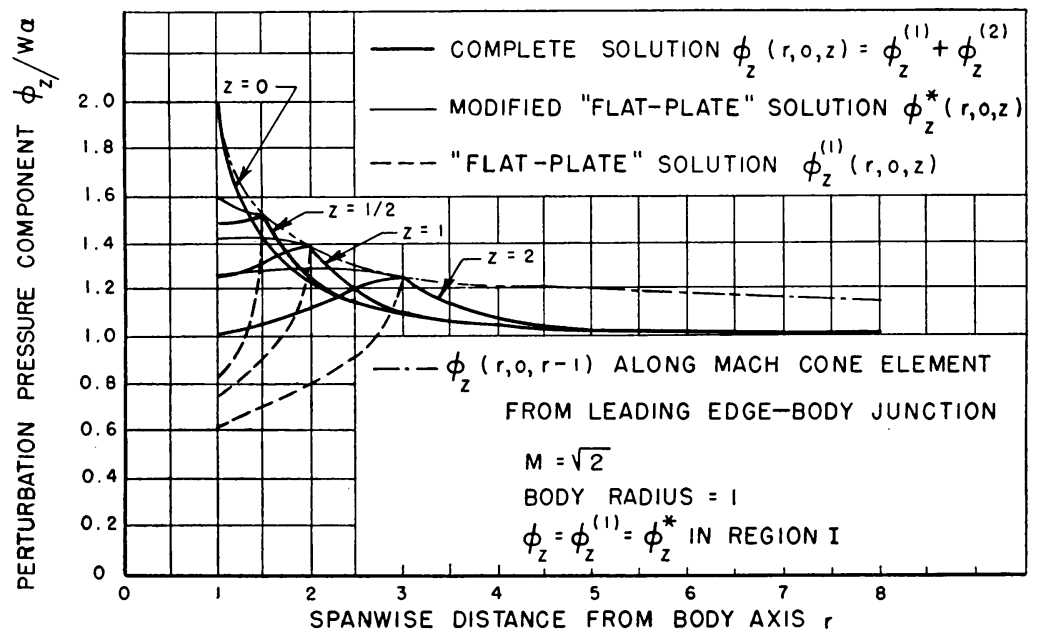

A) SPANWISE

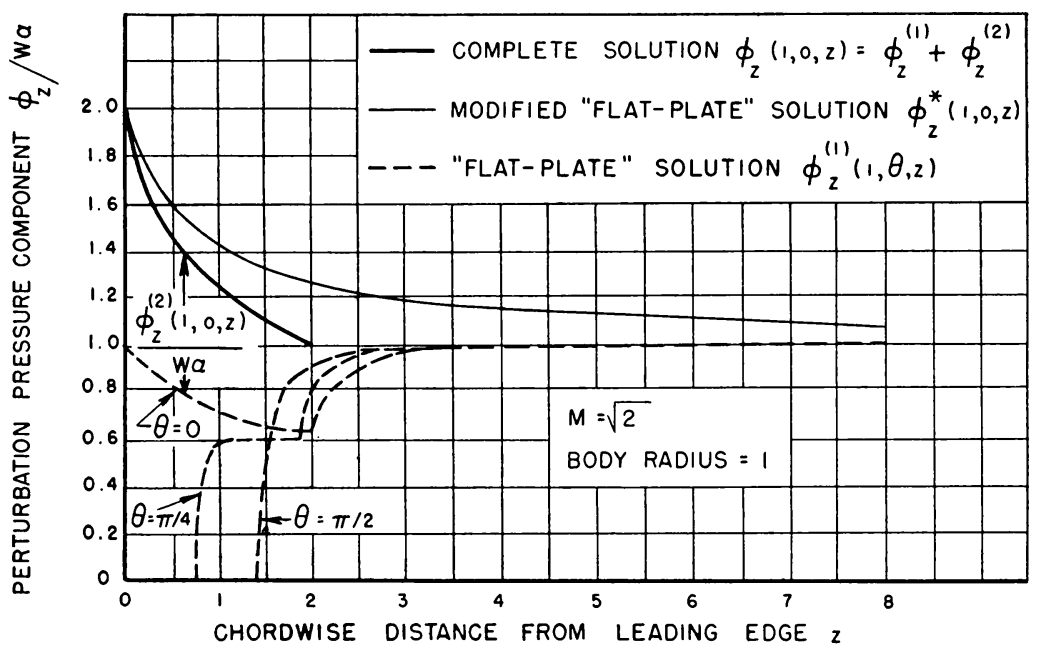

B) CHOROWISE

Fig. 4. Pressure Distribution on Surface: A) Spanwise, B) Chordwise.

plate" solution $\varphi^{*}$ ) can be interpreted as an upper bound (at least in Region II) to the complete solution. The pressure component is given by

$$
\begin{aligned}
\varphi_{z}^{*}(x, 0, z)=\frac{1}{\pi} \int_{1}^{(z+x)}\left(1+\frac{1}{\xi^{2}}\right)\left[z^{2}\right. & \left.-(x-\xi)^{2}\right]^{-1 / 2} d \xi \\
& +\frac{1}{\pi} \int_{1}^{(z-x+2)}\left(1+\frac{1}{\xi^{2}}\right)\left[z^{2}-(x-2+\xi)^{2}\right]^{-1 / 2} d \xi
\end{aligned}
$$


and is given in Fig. 4 by the light solid lines. Note that the first integral is the "flat plate" solution in Region II (cf. JPL report) and the second integral can be interpreted as the correction solution needed to satisfy the boundary conditions of zero flow through the semi-infinite plane barrier (cf. interpretation of "body" solution in Sec. 3).

5. Remarks on the "body" solution $\varphi^{(2)}$. From the comparison of the "flat plate" and "modified flat plate" solutions in the previous section and the discussion on the splitting of the complete solution in Section 3, it is evident that the "body" solution, in terms of the pressure component $\varphi_{z}^{(2)}$, is effective only within the Mach cones from the wing leading edge-body junction and is identically zero outside this region. The inverse Laplace transform required in the particular case of pressure on the body, $r=1$, is

$$
\mathcal{L}^{-1}\left\{\frac{K_{2 n}\left(s r^{\prime}\right)}{s K_{2 n}^{\prime}(s)} ; z\right\} \equiv \frac{1}{2 \pi i} \int_{\Gamma} e^{s z}\left[\frac{K_{2 n}\left(s r^{\prime}\right)}{s K_{2 n}(s)}\right] d s, \quad r^{\prime} \geq 1, n \geq 1,
$$

where the contour $\Gamma$ goes from $(a-i \cdot \infty)$ to $(a+i \cdot \infty)$, a real, $a>0$ in the complex $s$-plane. By a well-known procedure, Eq. (12) leads to

$$
\begin{aligned}
\mathscr{L}^{-1}\left\{\frac{K_{2 n}\left(s r^{\prime}\right)}{s K_{2 n}(s)} ; z\right\}=\int_{0}^{\infty} e^{-z \sigma}\left\{\frac{K_{2 n}\left(\sigma r^{\prime}\right) I_{2 n}^{\prime}(\sigma)-K_{2 n}^{\prime}(\sigma) I_{2 n}\left(\sigma r^{\prime}\right)}{\left[K_{2 n}^{\prime}(\sigma)\right]^{2}+\pi^{2}\left[I_{2 n}(\sigma)\right]^{2}}\right\} & \frac{d \sigma}{\sigma} \\
& +\sum_{m=1}^{n} \operatorname{Res} \cdot\left\{\frac{K_{2 m}\left(s r^{\prime}\right)}{s K_{2 m}^{\prime}(s)}\right\}
\end{aligned}
$$

The integral probably can be evaluated only by numerical methods, and the residues of the function (although the singularities, which are in the left-half $s$-plane, are simple poles) can be evaluated only after determining the location and number (which are of the order of $2^{n}$ ) of poles. The calculation complexity is then clear, even for this particular case, $r=1$, and an approximate method of solution is sought.

6. An approximate "body" solution $\varphi^{(2)}$ and the complete solution $\varphi$. The comparison between the "flat plate" and "modified flat plate" solutions implies that the main contribution to the pressure of the "body" solution will be found near the leading edgebody junction, i.e., for small $z$. With this in mind, an approximation for large $s$ to the Green's function, $G_{2}(P ; Q)$, given by Eq. (7e) for the region exterior to the unit circle is constructed. Consider a Green's function of the form

$$
\begin{aligned}
G\left(r, \theta ; r^{\prime}, \theta^{\prime}\right)=K_{0}\left[s \left(r^{2}+r^{\prime 2}-2 r r^{\prime}\right.\right. & \left.\left.\cos \left(\theta-\theta^{\prime}\right)\right)^{1 / 2}\right] \\
& +A K_{0}\left[s\left(r^{2}+\frac{1}{r^{\prime 2}}-\frac{2 r}{r^{\prime}} \cos \left(\theta-\theta^{\prime}\right)\right)^{1 / 2}\right],
\end{aligned}
$$

where $A$ is to be determined. It is clear, from the invariance properties of the differential equation (cf. Sec. 2), that the exact Green's function cannot be put in this form, since the second function on the right side of Eq. (13a) is the fundamental solution (with respect to $P(r, \theta)$ ) placed at the inverse point of $Q\left(r^{\prime}, \theta^{\prime}\right)$ with respect to the unit circle. But, by the addition formula (cf. Eq. $7 \mathrm{~b}$ ),

$$
\begin{aligned}
& K_{0}\left[s\left(r^{2}+\frac{1}{r^{\prime 2}}-\frac{2 r}{r^{\prime}} \cos \left(\theta-\theta^{\prime}\right)\right)^{1 / 2}\right]=K_{0}(s r) I_{0}\left(\frac{s}{r^{\prime}}\right) \\
&+2 \sum_{n=1}^{\infty} K_{n}(s r) I_{n}\left(\frac{s}{r^{\prime}}\right) \cos \left[n\left(\theta-\theta^{\prime}\right)\right], \quad r>\frac{1}{r^{\prime}}
\end{aligned}
$$


Replacing this expression in Eq. (13a) and comparing with the exact Green's function (Eq. 7e), such that the first term is exactly matched, the arbitrary function $A$ becomes

$$
A=-\frac{I_{0}^{\prime}(s)}{K_{0}^{\prime}(s)} \cdot \frac{K_{0}\left(s r^{\prime}\right)}{I_{0}\left(\frac{s}{r^{\prime}}\right)} .
$$

Then the approximate Green's function for the region exterior to the unit circle is

$$
\begin{aligned}
G_{2}\left(r, \theta ; r^{\prime}, \theta^{\prime}\right)=K_{0}\left[s \left(r^{2}+\right.\right. & \left.\left.r^{\prime 2}-2 r r^{\prime} \cos \left(\theta-\theta^{\prime}\right)\right)^{1 / 2}\right] \\
& +\frac{I_{0}^{\prime}(s)}{K_{0}^{\prime}(s)} \frac{K_{0}\left(s r^{\prime}\right)}{I_{0}\left(s / r^{\prime}\right)} K_{0}\left[s\left(r^{2}+\frac{1}{r^{\prime 2}}-\frac{2 r}{r^{\prime}} \cos \left(\theta-\theta^{\prime}\right)\right)^{1 / 2}\right]
\end{aligned}
$$

This approximate Green's function satisfies the differential equation, Eq. (2a), with respect to the point $P(r, \theta)$, but it is not symmetric in $\left(r, \theta ; r^{\prime}, \theta^{\prime}\right)$ and does not satisfy the boundary conditions. However, comparing term by term, the difference in the Green's functions occurs only under the summation sign, where

$$
\frac{I_{n}^{\prime}(s)}{K_{n}^{\prime}(s)} K_{n}(s r) K_{n}\left(s r^{\prime}\right) \cos \left[n\left(\theta-\theta^{\prime}\right)\right]
$$

in the exact Green's function (Eq. 7e) has been replaced by

$$
\frac{I_{0}^{\prime}(s)}{K_{0}^{\prime}(s)} \frac{I_{n}\left(s / r^{\prime}\right)}{I_{0}\left(s / r^{\prime}\right)} K_{n}(s r) K_{n}\left(s r^{\prime}\right) \cos \left[n\left(\theta-\theta^{\prime}\right)\right]
$$

in the approximate Green's function. Then for fixed $r^{\prime}$ and large $s$ (for Re $s>0$ ) terms given by Eq. (13f) approach the exact terms given by Eq. (13e), using the asymptotic expansions for the modified Bessel function [11].

The approximate Green's function for the region and boundary for the wing-body problem (Fig. 2) can be written immediately

$$
\begin{aligned}
G\left(r, \theta ; r^{\prime}, \theta^{\prime}\right)=\left\{K_{0}\left[s\left(r^{2}+r^{\prime 2}-2 r r^{\prime} \cos \left(\theta-\theta^{\prime}\right)\right)^{1 / 2}\right]\right. \\
\left.+K_{0}\left[s\left(r^{2}+r^{\prime 2}-2 r r^{\prime} \cos \left(\theta+\theta^{\prime}\right)\right)^{1 / 2}\right]\right\} \\
+-\frac{I_{0}^{\prime}(s)}{K_{0}^{\prime}(s)} \frac{K_{0}\left(s r^{\prime}\right)}{I_{0}\left(s / r^{\prime}\right)}\left\{K_{0}\left[s\left(r^{2}+\frac{1}{r^{\prime 2}}-\frac{2 r}{r^{\prime}} \cos \left(\theta-\theta^{\prime}\right)\right)^{1 / 2}\right]\right. \\
\left.+K_{0}\left[s\left(r^{2}+\frac{1}{r^{\prime 2}}-\frac{2 r}{r^{\prime}} \cos \left(\theta+\theta^{\prime}\right)\right)^{1 / 2}\right]\right\} .
\end{aligned}
$$

The approximate Green's function is a good representation for large $s$ (and $r^{\prime} \geq 1$ ); this result implies, by a known theorem in Laplace transform theory [13], that the solution obtained by using this Green's function is a good approximation near the leading edge-body junction. The transformed approximate "body" solution is

$$
\begin{array}{r}
s \psi^{(2)}(r, \theta ; s)=\frac{W \alpha}{\pi} \int_{1}^{\infty}\left(1+\frac{1}{r^{\prime 2}}\right) \frac{I_{0}^{\prime}(s)}{K_{0}^{\prime}(s)} \frac{K_{0}\left(s r^{\prime}\right)}{I_{0}\left(s / r^{\prime}\right)}\left\{K_{0}\left[s\left(r^{2}+\frac{1}{r^{\prime 2}}-\frac{2 r}{r^{\prime}} \cos \theta\right)^{1 / 2}\right]\right. \\
\left.+K_{0}\left[s\left(r^{2}+\frac{1}{r^{\prime 2}}+\frac{2 r}{r^{\prime}} \cos \theta\right)^{1 / 2}\right]\right\} d r^{\prime}
\end{array}
$$


The second term in the braces of Eq. (14a) corresponds to the reflected solution with respect to the $y, z$ plane (cf. "flat plate" solution, Eq. 9c). Now the solution for the pressure component, $\varphi_{z}^{(2)}$, can be obtained in Region II (cf. Fig. 3) where the approximation is best (the reflected solution does not enter here). By the asymptotic expansions for the modified Bessel functions

$$
-\frac{I_{0}^{\prime}(s)}{K_{0}^{\prime}(s)} \frac{K_{0}\left(s r^{\prime}\right)}{I_{0}\left(s / r^{\prime}\right)} \sim \frac{1}{r^{\prime}} \exp \left\{-\frac{s\left(r^{\prime}-1\right)^{2}}{r^{\prime}}\right\}\left\{1+O\left(\frac{1}{s}\right)\right\} .
$$

Neglecting the higher order terms

$$
\begin{aligned}
-\frac{I_{0}^{\prime}(s)}{K_{0}^{\prime}(s)} \frac{K_{0}\left(s r^{\prime}\right)}{I_{0}\left(s / r^{\prime}\right)} K_{0}\left[s \left(r^{2}\right.\right. & \left.\left.+\frac{1}{r^{\prime 2}}-\frac{2 r}{r^{\prime}} \cos \theta\right)^{1 / 2}\right] \\
& \sim \frac{1}{r^{\prime}} \exp \left\{-\frac{s\left(r^{\prime}-1\right)^{2}}{r^{\prime}}\right\} K_{0}\left[s\left(r^{2}+\frac{1}{r^{\prime 2}}-\frac{2 r}{r^{\prime}} \cos \theta\right)^{1 / 2}\right] .
\end{aligned}
$$

For simplicity, the solution is carried out for $\theta=0$, i.e., in the plane of the wing only. Applying the inverse Laplace transformation to the right side of Eq. (14c)

$$
\begin{gathered}
\mathcal{L}^{-1}\left\{\frac{1}{r^{\prime}} \exp \left\{-\frac{s\left(r^{\prime}-1\right)^{2}}{r^{\prime}}\right\} K_{0}\left(s\left|r-\frac{1}{r^{\prime}}\right|\right) ; z\right\}=0, \quad r^{\prime}>(z-r+2), \\
=\left\{\left[z r^{\prime}-\left(r^{\prime}-1\right)^{2}\right]^{2}-\left(r r^{\prime}-1\right)^{2}\right\}^{-1 / 2}, \quad 1<r^{\prime}<(z-r+2) .
\end{gathered}
$$

Then the approximate "body" solution in Region II is

$$
\frac{\varphi_{z}^{(2)}(r, 0, z)}{W \alpha}=\frac{1}{\pi} \int_{1}^{(z-r+2)}\left(1+\frac{1}{r^{\prime 2}}\right)\left\{\left[z r^{\prime}-\left(r^{\prime}-1\right)^{2}\right]^{2}-\left(r r^{\prime}-1\right)^{2}\right\}^{-1 / 2} d r^{\prime} .
$$

As is to be expected, the integral vanishes for $(r-z)=1, z \neq 0$. This is an elliptictype integral which can be expressed in terms of the standard complete and incomplete elliptic integrals of the first and third kinds by known methods of reduction [14]. For example, in the particular case $r=1$, Eq. (15a) reduces to

$$
\frac{\varphi_{z}^{(2)}(1,0, z)}{W \alpha}=\frac{1}{\pi} \int_{0}^{1}\left[1+(1+z \tau)^{-2}\right]\left\{(1-\tau)(1+z \tau)\left[1+(1+z) \tau-z \tau^{2}\right]\right\}^{-1 / 2} d \tau .
$$

The complete approximate solution, $\varphi_{z}^{(1)}+\varphi_{z}^{(2)}$, is shown in Fig. 4 as heavy, solid lines. This solution also approaches the correct asymptotic value for large $z$, i.e., $\varphi_{z} / W \alpha=1$. No estimate has been made for the error, although in the JPL report such an estimate is indicated for a simpler problem, using the same approximate Green's function for the circle (Eq. 13d). Also, additional calculations on the body have not been made; however, it is clear that the complete solution approaches the correct asymptotic value very rapidly in the downstream direction on the body and in the vicinity of the body on the wing.*

${ }^{*} \mathrm{~A}$ comparison with experimental pressure distributions is made in [15] and results, obtained by integrating the pressure over finite wings (including cases with swept-back leading edges), have been used in [16] on supersonic wing-body lift. 
An estimate of the complete solution on the body $(r=1)$ for large $z$ is readily made. From Eq. (9b), setting $r=1$, the Green's function for small $s$ becomes

$$
\left\{\frac{K_{0}\left(s r^{\prime}\right)}{K_{0}^{\prime}(s)}+2 \sum_{n=1}^{\infty} \frac{K_{2 n}\left(s r^{\prime}\right)}{K_{2 n}^{\prime}(s)} \cos 2 n \theta\right\}=-s\left[K_{0}\left(s r^{\prime}\right)+2 \sum_{n=1}^{\infty} \frac{\cos 2 n \theta}{2 n r^{\prime 2 n}}\right] .
$$

Since $K_{0}\left(s r^{\prime}\right)$ is the dominant term for small $s(\operatorname{Re} s>0)$, the transformed complete solution becomes

$$
\frac{s \psi(1, \theta ; s)}{W \alpha}=\frac{2}{\pi} \int_{1}^{\infty}\left(1+\frac{1}{r^{\prime 2}}\right) K_{0}\left(s r^{\prime}\right) d r^{\prime} .
$$

Taking the inverse Laplace transform of $K_{0}\left(s r^{\prime}\right)$, the pressure component

$$
\frac{\varphi_{z}(1, \theta, z)}{W \alpha}=\frac{2}{\pi} \int_{1}^{z}\left(1+\frac{1}{r^{\prime 2}}\right)\left(z^{2}-{r^{\prime}}^{2}\right)^{-1 / 2} d r^{\prime}=1+\frac{2}{\pi} \frac{\left(z^{2}-1\right)^{1 / 2}}{z^{2}}
$$

and

$$
\lim _{z \rightarrow \infty}\left\{\frac{\varphi_{z}}{W \alpha} \frac{(1, \theta, z)}{W \alpha}\right\}=1
$$

\section{REFERENCES}

1. J. R. Spreiter, Aerodynamic properties of slender wing-body combinations at subsonic, transonic, and supersonic speeds, NACA TN No. 1662 (1948).

2. S. H. Browne, L. Friedman, and I. Hodes, A wing-body problem in a supersonic conical flow, North American Aviation Report No. AL-387 (1947).

3. C. Ferrari, Interference between wing and body at supersonic speeds-theory and numerical applications, J. Aero. Sc. 15, 317-336 (1948).

- Interference between wing and body at supersonic speeds-note on wind-tunnel results and addendum to calculations, J. Aero. Sc. 16, 542-546 (1949).

4. P. A. Lagerstrom and M. D. Van Dyke, General considerations about planar and non-planar lifting systems, Douglas Aircraft Report No. SM-13432 (1949).

5. W. D. Hayes, Linearized supersonic flow, North American Aviation Report No. AL-222 (1947).

6. J. C. Gunn, Linearized supersonic airfoil theory, Phil. Trans. Roy. Soc. London (A) 240, 327-373 (1947).

7. G. N. Ward, The approximate external flow past a quasi-cylindrical tube moving at supersonic speeds, Quarterly J. Mech. Appl. Math. 1, 225-245 (1948).

8. A. Sommerfeld, Partial differential equations in physics. Academic Press, New York, 1949.

9. B. B. Baker and E. T. Copson, The mathematical theory of Huygen's principle, Oxford University Press, London, 1939.

10. O. D. Kellogg, Foundations of potential theory, Springer, Berlin, 1929.

11. G. N. Watson, Theory of Bessel functions, Cambridge University Press, London, 1944.

12. A. Sommerfeld, Die Greensche Funktion der Schwingungsgleichung, Jahresber. Deutsch. Math. Ver. 21, 309-353 (1912).

13. J. C. Jeager and H. S. Carslaw, Operational methods in applied mathematics, Oxford University Press, London, 1947.

14. T. M. MacRobert, Functions of a complex variable, MacMillan and Co., London, 1933.

15. G. K. Morikawa, On wing-body interference at supersonic speeds, J. of Aero. Sc., 17, 315-316 (1950).

16. G. K. Morikawa, Supersonic wing-body lift, J. of Aero. Sc. 18, 217-228 (1951). 